\title{
On sgoldstino interpretation of HyperCP events
}

\author{
D. S. Gorbunov*, V. A. Rubakov ${ }^{\dagger}$ \\ Institute for Nuclear Research of the Russian Academy of Sciences, \\ 60th October Anniversary prospect 7a, Moscow 117312, Russia
}

\begin{abstract}
We discuss possible sgoldstino interpretation of the observation, reported by HyperCP collaboration, of three $\Sigma^{+} \rightarrow p \mu^{+} \mu^{-}$decay events with dimuon invariant mass $214.3 \mathrm{MeV}$ within detector resolution. We find that this interpretation is consistent in models with (i) parity conservation in sgoldstino interactions, (ii) pseudoscalar sgoldstino $P$ of mass $214 \mathrm{MeV}$ and heavier scalar sgoldstino, and (iii) low supersymmetry breaking scale, $\sqrt{F}=2.5-60 \mathrm{TeV}$. In these models, sgoldstino can be observed in decays $K^{+} \rightarrow \pi^{+} \pi^{0} P, K_{L} \rightarrow \pi \pi P$ and $K_{S} \rightarrow \pi \pi P$ at the branching ratios of at least of order $10^{-12}, 10^{-8}$ and $10^{-11}$, respectively. The model can be probed also at $e^{+} e^{-}$colliders in the channels $e^{+} e^{-} \rightarrow \gamma P$ and $e^{+} e^{-} \rightarrow e^{+} e^{-} P$, where the cross section is likely to be in the range $1 \mathrm{pb}-5$ ab.
\end{abstract}

\section{Introduction}

Recently HyperCP collaboration has reported [1] the first evidence for the decay $\Sigma^{+} \rightarrow p \mu^{+} \mu^{-}$with branching ratio at the level of $10^{-7}-10^{-8}$. The central value of the measured branching ratio is larger than most Standard Model (SM) estimates, although discrepancy with SM in the overall branching ratio may not be dramatic in view of large uncertainties in the hadronic

*e-mail: gorby@ms2.inr.ac.ru

†e-mail: rubakov@ms2.inr.ac.ru 
matrix elements [2]. The most remarkable property of the HyperCP events is that dimuon masses of all three of them are equal within the resolution of the detector. This suggests [1] that the observed $\mu^{+} \mu^{-}$pairs are decay products of a new neutral particle $X$ born in the hyperon decay

$$
\Sigma \longrightarrow p+X, \quad X \longrightarrow \mu^{+} \mu^{-}
$$

with branching ratio

$$
\operatorname{Br}\left(\Sigma \rightarrow p X\left(X \rightarrow \mu^{+} \mu^{-}\right)\right)=\left[3.1_{-1.9}^{+2.4}(\text { stat }) \pm 1.5(\text { syst })\right] \times 10^{-8},
$$

and mass of the intermediate neutral state $X$ equal to

$$
m_{X}=214.3 \pm 0.5 \mathrm{MeV} \text {. }
$$

As a phenomenologically viable explanation, supersymmetric models with light sgoldstino and parity conservation [3] have been proposed [1, 4], the state $X$ being interpreted as spinless particle $P$ (sgoldstino) — one of the superpartners of goldstino.

This interpretation is further discussed in this paper. Our purpose is to estimate the relevant range of model parameters and to discuss other experiments sensitive to such models.

From HyperCP results we will estimate the strength of sgoldstino coupling to muons, responsible for the sgoldstino decay $X \rightarrow \mu^{+} \mu^{-}$, and sgoldstino flavor violating coupling to $d$ - and $s$-quarks, responsible for the hyperon decay (1). In particular, we will find that the sgoldstino explanation of HyperCP events implies the scale of supersymmetry breaking $\sqrt{F}$ within the range 2.5-60 TeV (depending on hierarchy between MSSM soft terms $M_{\text {soft }}$ and $\sqrt{F}$ ). The estimate of the flavor violating coupling suggests that sgoldstino would show up in kaon physics. The observed events cannot be attributed to scalar sgoldstino, since in that case sgoldstino had to be already detected in two-body kaon decays. It is the pseudoscalar sgoldstino $P$ that can play a role of $\mathrm{X}$-particle.

Pseudoscalar sgoldstino $P$, responsible for the HyperCP events, can be observed in the decays $K^{+} \rightarrow \pi^{+} \pi^{0} P, K_{L} \rightarrow \pi \pi P$ and $K_{S} \rightarrow \pi \pi P$ at the branching ratios of at least of order $10^{-12}, 10^{-8}$ and $10^{-11}$, respectively. With the inferred value of $\sqrt{F}$, the model can be probed also at $e^{+} e^{-}$colliders in the channel $e^{+} e^{-} \rightarrow \gamma P$ and $e^{+} e^{-} \rightarrow e^{+} e^{-} P$, where the cross section is estimated as $1 \mathrm{pb}-5$ ab depending on hierarchy of MSSM soft terms and 
the value of $\sqrt{F}$. Finally, sgoldstino may be searched for via its resonant production in $e^{+} e^{-}$collision at $\sqrt{s}=214.3 \mathrm{MeV}$.

The paper is organized as follows. In Sec.2 supersymmetric models with light sgoldstino are briefly discussed and useful notations are introduced. The range of parameters, relevant for the explanation of HyperCP events, is estimated in Sec.3. In Sec.4 we study prospects of various experiments in testing sgoldstino couplings. Sec.5 contains concluding remarks.

\section{Models with light sgoldstino}

Superpartners of goldstino - longitudinal component of gravitino - may be fairly light. In a variety of models with low energy supersymmetry they are lighter than a few GeV. Such pattern emerges in a number of non-minimal supergravity models $[5,6]$ and also in gauge mediation models if supersymmetry is broken via non-trivial superpotential (see, e.g., Ref. [7] and references therein). Here we merely assume that sgoldstino masses are small, so that $\Sigma^{+}$-hyperon decay into proton and sgoldstino is kinematically allowed.

Sgoldstinos couple to MSSM fields in the same way as goldstino [8, 9], with corresponding coupling constants being proportional to the ratios of MSSM soft terms (squark and gaugino masses, trilinear coupling constants) to the supersymmetry breaking parameter $F$ (which is of the order of squared scale of supersymmetry breaking in the underlying theory). Constraints on sgoldstino couplings may be translated into the limits on $\sqrt{F}$. The most sensitive probes of sgoldstinos are flavor violating processes [10], provided that flavor is violated in squark and/or slepton sector. A sketch of the sgoldstino interactions, experimental constraints on the models with light sgoldstino and relevant references can be found in Refs. [10, 9].

A special class of supersymmetric extensions of the Standard Model in which interactions of sgoldstinos with quarks and gluons conserve parity but do not conserve quark flavor, has been considered in Ref. [3]. Parity conservation in sgoldstino interactions with quarks and gluons (as well as with leptons and photons) may not be accidental. As an example, it is natural in theories with spontaneously broken left-right symmetry (see Ref. [3] for details), which not only are aesthetically appealing but also provide a solution [11] to the strong CP-problem.

It was found [3] that if the pseudoscalar sgoldstino $P$ is light, $m_{P}<$ $\left(m_{K}-2 m_{\pi}\right)$, and the scalar sgoldstino is heavier, $m_{S}>\left(m_{K}-m_{\pi}\right)$, an 
interesting place for experimental searches is the poorly explored area of three-body decays of kaons, $K_{S, L}^{0} \rightarrow \pi^{+} \pi^{-} P, K_{S, L}^{0} \rightarrow \pi^{0} \pi^{0} P$ and $K^{+} \rightarrow$ $\pi^{+} \pi^{0} P$, with $P$ subsequently decaying into $\gamma \gamma$, possibly $e^{+} e^{-}, \mu^{+} \mu^{-}$, or flying away from the detector. Possible sgoldstino contribution to hyperon physics has been considered in Ref. [12]. It was shown that searches for hyperon decays into baryon and sgoldstino are very sensitive to sgoldstino couplings in models with light pseudoscalar sgoldstino, $m_{P}<\left(m_{\Sigma}-m_{p}\right)$, heavy scalar sgoldstino, $m_{S}>\left(m_{K}-m_{\pi}\right)$, and parity conservation. In what follows we will consider models with parity conservation in sgoldstino interactions, and make a remark on the parity-violating models in an appropriate place.

We are interested in trilinear couplings between sgoldstino and SM fields, which can be derived by making use of spurion technique (see e.g., Refs. [13, $9]$ ). The coupling constants of sgoldstino to $S U(3)_{c} \times S U(2)_{\mathrm{W}} \times U(1)_{\mathrm{Y}}$ gauge bosons are proportional to the ratio of gaugino soft mass terms to vacuum expectation value of the auxiliary component $F$ of the spurion superfield, while the coupling constants of sgoldstino to SM fermions $f$ are proportional to the product of fermion mass $m_{f}$ and the ratio of trilinear soft term $A_{f}$ to $F$. In particular, relevant for our study flavor-blind sgoldstino interactions are determined by the following effective lagrangians [9]: for pseudoscalar sgoldstino $P$

$$
\mathcal{L}_{P \gamma \gamma}=\frac{M_{\gamma \gamma}}{4 \sqrt{2} F} P F_{\mu \nu} F_{\lambda \rho} \epsilon^{\mu \nu \lambda \rho}, \quad \mathcal{L}_{P \bar{f} f}=-i \frac{m_{f} A_{f}}{\sqrt{2} F} P \bar{f} \gamma_{5} f
$$

and for scalar sgoldstino $S$

$$
\mathcal{L}_{S \gamma \gamma}=-\frac{M_{\gamma \gamma}}{2 \sqrt{2} F} S F_{\mu \nu} F^{\mu \nu}, \quad \mathcal{L}_{S \bar{f} f}=-\frac{m_{f} A_{f}}{\sqrt{2} F} S \bar{f} f,
$$

where $M_{\gamma \gamma}=M_{1} \cos ^{2} \theta_{\mathrm{w}}+M_{2} \sin ^{2} \theta_{\mathrm{w}}$ with $M_{i}$ being gaugino masses. It is worth noting that in many models of supersymmetry breaking one has $M_{\gamma \gamma}, A_{f} \ll \sqrt{F}$, while the opposite case, $M_{\gamma \gamma}, A_{f} \sim \sqrt{F}$, corresponds to unitarity limit.

Of special interest here are also the couplings of sgoldstinos to $d$ - and $s$-quarks, which violate flavor. In models with parity conservation it is convenient to parameterize the corresponding interaction terms as [3]

$$
\begin{aligned}
& \mathcal{L}_{P d s}=-P \cdot\left(h_{12}^{(D)} \cdot \bar{d} i \gamma^{5} s+\text { h.c. }\right) \\
& \mathcal{L}_{S d s}=-S \cdot\left(h_{12}^{(D)} \cdot \bar{d} s+\text { h.c. }\right)
\end{aligned}
$$


where

$$
h_{12}^{(D)}=\frac{1}{\sqrt{2}} \frac{\tilde{m}_{D, 12}^{(L R) 2}}{F}
$$

and $\tilde{m}_{D, i j}^{(L R) 2}, i, j=1,2,3$ are left-right soft terms in the matrix of squared masses of squarks. These interaction terms are assumed to be responsible for the hyperon decay (1).

\section{Model parameters from HyperCP results}

Couplings (5) and (6) give rise to the hyperon decays [12]

$$
\Sigma^{+} \rightarrow p P
$$

and

$$
\Sigma^{+} \rightarrow p S
$$

respectively, whose rates can be estimated by making use of the matrix elements

$$
\begin{aligned}
\left\langle p\left|\bar{s} \gamma^{\mu} \gamma_{5} d\right| \Sigma^{+}\right\rangle & =B \cdot \bar{u}_{p} \gamma^{\mu} \gamma_{5} u_{\Sigma} \\
\left\langle p\left|\bar{s} \gamma^{\mu} d\right| \Sigma^{+}\right\rangle & =A \cdot \bar{u}_{p} \gamma^{\mu} u_{\Sigma}
\end{aligned}
$$

and neglecting the external momentum dependence of the form factors $A$ and $B$. The isospin symmetry implies that these constants are the same as ones describing $\Sigma^{-} \rightarrow n e^{-} \nu$ decay, hence [14],

$$
A=1, \quad B=0.34 \text {. }
$$

Then

$$
\begin{aligned}
\left\langle p\left|\bar{s} \gamma_{5} d\right| \Sigma^{+}\right\rangle & =-B \frac{m_{\Sigma}+m_{p}}{m_{s}} \cdot \bar{u}_{p} \gamma_{5} u_{\Sigma}, \\
\left\langle p|\bar{s} d| \Sigma^{+}\right\rangle & =A \frac{m_{\Sigma}-m_{p}}{m_{s}} \cdot \bar{u}_{p} u_{\Sigma},
\end{aligned}
$$

where $m_{s}, m_{\Sigma}$ and $m_{p}$ are masses of $s$-quark, $\Sigma$-hyperon and proton, respectively. These amplitudes yield the hyperon decay rates [12]

$$
\begin{aligned}
& \Gamma_{\Sigma^{+} \rightarrow p P}=\frac{\left|h_{12}^{(D)}\right|^{2}|B|^{2}}{8 \pi} \frac{\left(m_{\Sigma}+m_{p}\right)^{2}}{m_{s}^{2}}\left(\left(1-\frac{m_{p}}{m_{\Sigma}}\right)^{2}-\frac{m_{P}^{2}}{m_{\Sigma}^{2}}\right) \cdot q_{P}, \\
& \Gamma_{\Sigma^{+} \rightarrow p S}=\frac{\left|h_{12}^{(D)}\right|^{2}|A|^{2}}{8 \pi} \frac{\left(m_{\Sigma}-m_{p}\right)^{2}}{m_{s}^{2}}\left(\left(1+\frac{m_{p}}{m_{\Sigma}}\right)^{2}-\frac{m_{S}^{2}}{m_{\Sigma}^{2}}\right) \cdot q_{S},
\end{aligned}
$$


where

$$
q_{X}=\frac{1}{2 m_{\Sigma}} \sqrt{\left(\left(m_{\Sigma}+m_{X}\right)^{2}-m_{p}^{2}\right)\left(\left(m_{\Sigma}-m_{X}\right)^{2}-m_{p}^{2}\right)}, \quad X=S, P .
$$

Let us turn to the results of HyperCP collaboration [1]. Considering the central values of Eqs. (2), (3) as a reference point, one obtains from Eqs. (7), (8)

$$
\begin{aligned}
\left|h_{12}^{(D)}\right| \mathrm{Br}^{1 / 2}\left(P \rightarrow \mu^{+} \mu^{-}\right) & =3.8 \cdot 10^{-10}, \\
\left|h_{12}^{(D)}\right| \operatorname{Br}^{1 / 2}\left(S \rightarrow \mu^{+} \mu^{-}\right) & =6.8 \cdot 10^{-11},
\end{aligned}
$$

for pseudoscalar and scalar sgoldstino, respectively. In models where scalar sgoldstino $S$ is light, the strongest limit on $\left|h_{12}^{(D)}\right|^{2} \operatorname{Br}\left(S \rightarrow \mu^{+} \mu^{-}\right)$comes from searches for two-body charged kaon decays $^{1}$ with subsequent sgoldstino decay into $\mu^{+} \mu^{-}$(see Ref. [10] for details),

$$
\left|h_{12}^{(D)}\right| \operatorname{Br}^{1 / 2}\left(S \rightarrow \mu^{+} \mu^{-}\right)<6 \cdot 10^{-12} .
$$

Thus, hyperon decay into proton and sgoldstino is highly suppressed in these models, and the anomalous events observed in HyperCP experiment cannot be attributed to light scalar sgoldstino. In models with light pseudoscalar sgoldstino and parity violating sgoldstino-quark couplings the bound similar to (10) applies to $\left|h_{12}^{(D)}\right|^{2} \operatorname{Br}\left(P \rightarrow \mu^{+} \mu^{-}\right)$; this bound excludes sgoldstino explanation of HyperCP result in those models. The remaining possibility, which is the focus of this paper, is pseudoscalar sgoldstino and models with parity conservation. Then, the strongest constraints [3] on $h_{12}^{(D)}$ come from the study of $K^{0}-\bar{K}^{0}$ system and searches for $K \rightarrow \pi \pi P$ decays. The corresponding limits on $h_{12}^{(D)}$ are well above the value (9), hence the HyperCP events can indeed be explained by pseudoscalar sgoldstino.

HyperCP collaboration did not present any estimate of $X$-particle lifetime, $\tau_{\mathrm{x}}$, since the opening angles of dimuons are small and vertex resolution along the beam axis is rather poor. Using reasonable estimates for the HyperCP decay vertex resolution (about 2 meters, see Ref. [17]) and measured $\gamma$-factors of the muons of anomalous events (about 270), one can place the upper limit

$$
\tau_{X} \lesssim 2.5 \cdot 10^{-11} \mathrm{~s}
$$

\footnotetext{
${ }^{1}$ The bound (10) is independent of the phase of $h_{12}^{(D)}$. Even stronger bound [15] is obtained from the search for $K_{L} \rightarrow \pi S\left(S \rightarrow \mu^{+} \mu^{-}\right)$decay [16]. The latter bound is valid, however, for $\operatorname{Re}\left[h_{12}^{(D)}\right] \simeq\left|h_{12}^{(D)}\right|$ only, and does not apply in the case of $\operatorname{Re}\left[h_{12}^{(D)}\right]=0$.
} 
We note in passing that the above considerations in this section apply to any pseudoscalar and scalar particles with couplings (5) and (6).

For sgoldstino of mass (3), only decay channels into photons, $e^{+} e^{-}, \mu^{+} \mu^{-}$ and, possibly, gravitinos, contribute to the sgoldstino total width. Since sgoldstino-fermion couplings (4) are proportional to the fermion masses, the contribution of $e^{+} e^{-}$is negligible (we will comment on the possible opposite case in Sec.4). The contribution of invisible mode (decay into gravitinos), which is suppressed by $m_{P}^{2} / M_{\text {soft }}^{2}$ (see e.g. Ref. [10] for details) is also negligible. Thus, sgoldstino width $\Gamma_{P}$ is saturated by two contributions,

$$
\Gamma(P \rightarrow \gamma \gamma)=\frac{m_{P}^{3} M_{\gamma \gamma}^{2}}{32 \pi F^{2}}, \quad \Gamma(P \rightarrow \mu \bar{\mu})=\frac{m_{P} m_{\mu}^{2} A_{\mu}^{2}}{16 \pi F^{2}}\left(1-\frac{4 m_{\mu}^{2}}{m_{P}^{2}}\right)^{1 / 2} .
$$

At $M_{\gamma \gamma} \sim A_{\mu}$ the latter contribution is suppressed by an order of magnitude mainly due to the phase space factor. Thus, without strong hierarchy between MSSM soft terms, sgoldstino of mass (3) decays predominantly into two photons. Unfortunately, HyperCP had no $\gamma$-detector, that prevented the cross check of the sgoldstino explanation of the anomalous events. ${ }^{2}$

What can be estimated from Eqs. (11) and (12) is the upper limit on the scale of supersymmetry breaking $\sqrt{F}$. At $M_{\gamma \gamma}=A_{\mu}=100 \mathrm{GeV}$ one has $\sqrt{F} \simeq 2.5 \mathrm{TeV}$, while in the the unitarity limit, $M_{\gamma \gamma} \sim A_{\mu} \sim \sqrt{F}$, one finds $\sqrt{F} \simeq 60 \mathrm{TeV}$. Hence, sgoldstino explanation of HyperCP events definitely implies low energy scale of supersymmetry breaking.

We note that Eqs. (12) also imply a lower bound on sgoldstino lifetime. The shortest lifetime occurs in the unitarity limit $M_{\gamma \gamma} \sim \sqrt{F}$ and for $\sqrt{F}$ saturating the experimental bound $\sqrt{F} \sim 500 \mathrm{GeV}$. One has

$$
\tau_{P} \gtrsim 1.7 \cdot 10^{-15} \mathrm{~s}
$$

for $M_{P}=214.3 \mathrm{MeV}$.

\section{Possible experimental tests of sgoldstino ev- idence}

There are two independent direct tests of the sgoldstino explanation of HyperCP results. One is related to sgoldstino flavor violating coupling and

\footnotetext{
${ }^{2}$ Note that coupling (5) yields effective sgoldstino-neutron- $\Lambda$ interaction, but sgoldstino with $M_{P}=213.4 \mathrm{MeV}$ is too heavy to be searched for in $\Lambda$-hyperon decay. On the other hand, kinematically allowed is the decay $\Omega^{-} \rightarrow \Xi^{-}+P$.
} 
another one is related to sgoldstino flavor-blind couplings, whose strength has been estimated in the previous section.

Sgoldstino- $d$ - $s$ coupling gives rise to rare three-body kaon decays into two pions and sgoldstino, whose branching ratios can be estimated by making use of amplitudes presented in Appendix A with $h_{12}^{(D)}$ given by Eq. (9). The results for neutral kaons depend on the possible hierarchy between real and imaginary parts of $h_{12}^{(D)}$, which cannot be extracted from HyperCP data. One obtains

$$
\operatorname{Br}\left(K^{+} \rightarrow \pi^{+} \pi^{0} P\left(P \rightarrow \mu^{+} \mu^{-}\right)\right)=1.2 \cdot 10^{-12}, \text { any } \operatorname{Re}\left[h_{12}^{(D)}\right] / \operatorname{Im}\left[h_{12}^{(D)}\right] .
$$

For $\operatorname{Re}\left[h_{12}^{(D)}\right] \simeq 0$, decays of $K_{L}^{0}$ are suppressed,

$$
\operatorname{Br}\left(K_{L}^{0} \rightarrow \pi^{+} \pi^{-} P\left(P \rightarrow \mu^{+} \mu^{-}\right)\right) \simeq 3.0 \cdot 10^{-13}, \quad \operatorname{Re}\left[h_{12}^{(D)}\right]=0,
$$

while in the opposite case

$$
\begin{aligned}
\operatorname{Br}\left(K_{L}^{0} \rightarrow \pi^{+} \pi^{-} P\left(P \rightarrow \mu^{+} \mu^{-}\right)\right) \simeq 2.4 \cdot 10^{-9}, \quad \operatorname{Re}\left[h_{12}^{(D)}\right] \simeq\left|h_{12}^{(D)}\right| \\
\operatorname{Br}\left(K_{L}^{0} \rightarrow \pi^{0} \pi^{0} P\left(P \rightarrow \mu^{+} \mu^{-}\right)\right) \simeq 1.2 \cdot 10^{-8}, \quad \operatorname{Re}\left[h_{12}^{(D)}\right] \simeq\left|h_{12}^{(D)}\right| .
\end{aligned}
$$

On the other hand, decays of $K_{S}^{0}$ are suppressed at $\operatorname{Im}\left[h_{12}^{(D)}\right] \simeq 0$,

$$
\operatorname{Br}\left(K_{S}^{0} \rightarrow \pi^{+} \pi^{-} P\left(P \rightarrow \mu^{+} \mu^{-}\right)\right) \simeq 5.2 \cdot 10^{-16}, \quad \operatorname{Im}\left[h_{12}^{(D)}\right]=0,
$$

while without this hierarchy one has

$$
\begin{aligned}
& \operatorname{Br}\left(K_{S}^{0} \rightarrow \pi^{+} \pi^{-} P\left(P \rightarrow \mu^{+} \mu^{-}\right)\right) \simeq 4.1 \cdot 10^{-12}, \operatorname{Im}\left[h_{12}^{(D)}\right] \simeq\left|h_{12}^{(D)}\right|, \\
& \operatorname{Br}\left(K_{S}^{0} \rightarrow \pi^{0} \pi^{0} P\left(P \rightarrow \mu^{+} \mu^{-}\right)\right) \simeq 2.1 \cdot 10^{-11}, \quad \operatorname{Im}\left[h_{12}^{(D)}\right] \simeq\left|h_{12}^{(D)}\right| .
\end{aligned}
$$

These results are obtained to the leading order in chiral perturbation theory. One may expect that actual numbers may be $30 \%$ to $50 \%$ larger. Indeed, experimental data on the process $K^{+} \rightarrow \pi \pi e \nu$, which is quite similar to the decays we consider, are noticeable larger than the leading order predictions of chiral perturbation theory (see, e.g., Refs. [18, 19]).

For similar modes with sgoldstino decaying into photons one gets the same numbers multiplied by $\Gamma(P \rightarrow \gamma \gamma) / \Gamma\left(P \rightarrow \mu^{+} \mu^{-}\right)$. Therefore, the branching ratios of the decays $K \rightarrow \pi \pi P(P \rightarrow \gamma \gamma)$ may be substantially higher ${ }^{3}$. Note

\footnotetext{
${ }^{3}$ As an example, for $A_{\mu} \sim \alpha_{2} M_{\gamma \gamma}$ (which is still a fairly natural possibility), branching ratios of $K \rightarrow \pi \pi P(P \rightarrow \gamma \gamma)$ are larger than given in (14) - (20) by a factor of order $10^{4}$.
} 
that decays into final state with neutral pions have significantly higher rates, as compared to charged pions, because of larger phase space. Note also that the above estimates for the branching ratios of the decays (14) - (20) apply not only to sgoldstino, but to any pseudoscalar particle explaining the HyperCP events.

Flavor-blind sgoldstino-photon and sgoldstino-muon couplings can be tested at $e^{+} e^{-}$colliders. Sgoldstino coupling constants to leptons are dimensionless and proportional to lepton masses, hence the most promising processes of sgoldstino production involve sgoldstino-photon coupling. Sgoldstino can be searched for in $e^{+} e^{-} \rightarrow \gamma P$ (fig. 1a) and $e^{+} e^{-} \rightarrow e^{+} e^{-} P$ (fig. 1b) with

(a)

(b)

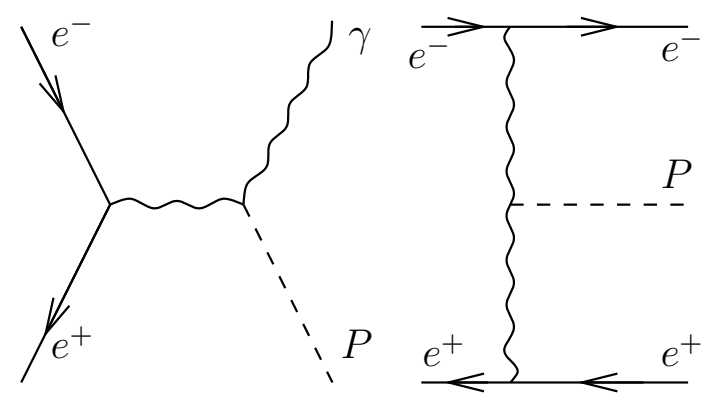

Figure 1: Amplitudes contributing to sgoldstino production in $e^{+} e^{-}$collisions; contributions to the same final states, associated with sgoldstinoelectron coupling, are suppressed.

sgoldstino subsequently decaying into a pair of photons or $\mu^{+} \mu^{-}$-pair.

The cross section of $P \gamma$ production is

$$
\begin{aligned}
\sigma_{P \gamma} & =\frac{\alpha}{12} \frac{M_{\gamma \gamma}^{2}}{F^{2}}\left(1-\frac{m_{P}^{2}}{s}\right)^{3} \\
& =\frac{8 \alpha \pi}{3} m_{P}^{-3}\left(1-\frac{m_{P}^{2}}{s}\right)^{3} \Gamma(P \rightarrow \gamma \gamma) .
\end{aligned}
$$

The sgoldstino signals in different channels depend on the pattern of sgoldstino branching ratios. If sgoldstino width is saturated by decay into photon 
pair, then, with account of (11) and (13), one finds from Eq. (21)

$$
\begin{aligned}
\sigma_{e^{+} e^{-} \rightarrow \gamma P(P \rightarrow \gamma \gamma)} & =\frac{\sigma_{P \gamma}}{\Gamma(P \rightarrow \gamma \gamma)} \frac{1}{\tau_{P}}=64 \mathrm{ab} \div 0.95 \mathrm{pb}, \\
\sigma_{e^{+} e^{-} \rightarrow \gamma P\left(P \rightarrow \mu^{+} \mu^{-}\right)} & =\frac{\sigma_{P \gamma}}{\Gamma(P \rightarrow \gamma \gamma)} \frac{1}{\tau_{P}} \frac{\Gamma\left(P \rightarrow \mu^{+} \mu^{-}\right)}{\Gamma(P \rightarrow \gamma \gamma)}=(5.2 \mathrm{ab} \div 77 \mathrm{fb}) \cdot \frac{A_{\mu}^{2}}{M_{\gamma \gamma}^{2}},
\end{aligned}
$$

where smaller and larger values refer to the lifetimes saturating (11) and (13), respectively. These formulae are valid for $A_{\mu} / M_{\gamma \gamma}<3.5$, when the two-photon decay mode is indeed dominant.

In the opposite case, with dominant decay into $\mu^{+} \mu^{-}$, one obtains

$$
\begin{aligned}
& \sigma_{e^{+} e^{-} \rightarrow \gamma P(P \rightarrow \gamma \gamma)}=\frac{\sigma_{P \gamma}}{\Gamma\left(P \rightarrow \mu^{+} \mu^{-}\right)} \frac{1}{\tau_{P}} \frac{\Gamma(P \rightarrow \gamma \gamma)}{\Gamma\left(P \rightarrow \mu^{+} \mu^{-}\right)}=(9.9 \mathrm{fb} \div 145 \mathrm{pb}) \cdot \frac{M_{\gamma \gamma}^{4}}{A_{\mu}^{4}}, \\
& \sigma_{e^{+} e^{-} \rightarrow \gamma P\left(P \rightarrow \mu^{+} \mu^{-}\right)}=\frac{\sigma_{P \gamma}}{\Gamma\left(P \rightarrow \mu^{+} \mu^{-}\right)} \frac{1}{\tau_{P}}=(0.8 \mathrm{fb} \div 12 \mathrm{pb}) \cdot \frac{M_{\gamma \gamma}^{2}}{A_{\mu}^{2}} .
\end{aligned}
$$

The latter formulae are relevant for $M_{\gamma \gamma} / A_{\mu}<0.28$, otherwise the assumption of dominant decay into $\mu^{+} \mu^{-}$would not be valid.

The cross section of $e^{+} e^{-} P$ production, with the accuracy $1 / \ln \frac{s}{m_{e}^{2}}$, is [14]

$$
\begin{aligned}
\sigma_{e^{+} e^{-P}} & =8 \alpha^{2} \frac{\Gamma(P \rightarrow \gamma \gamma)}{m_{P}^{3}} \cdot\left(f\left(m_{P}^{2} / s\right) \cdot \ln ^{2} \frac{s}{m_{e}^{2}}-\frac{1}{3} \ln ^{3} \frac{s}{m_{P}^{2}}\right), \\
f(z) & =\left(1+\frac{1}{2} z\right)^{2} \ln \frac{1}{z}-\frac{1}{2}(1-z)(3+z) .
\end{aligned}
$$

It grows with energy, becoming comparable with $\gamma P$ production at $\sqrt{s} \simeq$ $0.5 \mathrm{GeV}$ and exceeding $\gamma P$ cross section by a factor of 16 at $\sqrt{s} \simeq 10 \mathrm{GeV}$.

Another possibility of searching for light sgoldstino in $e^{+} e^{-}$-annihilation is sgoldstino resonant production with the beams' energy tuned to sgoldstino mass. In this case the cross section of sgoldstino production exactly at the resonance peak is

$$
\begin{aligned}
\sigma_{e^{+} e^{-} \rightarrow P} & =\frac{2 \pi^{2}}{m_{P}^{2}} \cdot \operatorname{Br}\left(P \rightarrow e^{+} e^{-}\right)= \\
& =167 \mathrm{mb} \cdot \operatorname{Br}\left(P \rightarrow e^{+} e^{-}\right) .
\end{aligned}
$$

If sgoldstino width is saturated by the decay into $\mu^{+} \mu^{-}$, then at the peak

$$
\sigma_{e^{+} e^{-} \rightarrow P}=24 \mu \mathrm{b} \cdot \frac{A_{e}^{2}}{A_{\mu}^{2}} .
$$


If the $\gamma \gamma$ mode dominates over $\mu^{+} \mu^{-}$, then

$$
\sigma_{e^{+} e^{-} \rightarrow P}=1.9 \mu \mathrm{b} \cdot \frac{A_{e}^{2}}{M_{\gamma \gamma}^{2}} .
$$

To estimate the number of events in realistic case one multiplies the above values by the product of collider luminosity and the ratio of the sgoldstino width to the beam energy spread. The latter is typically of order

$$
\frac{\Delta p}{p} \equiv \eta \cdot 10^{-3}
$$

with $\eta \sim 1$. Thus, the suppression factor is $\eta \cdot\left(1.2 \cdot 10^{-10}-1.8 \cdot 10^{-6}\right)$ for sgoldstino lifetimes saturating the bounds (11) and (13), respectively. Hence, in the case $A_{e}=A_{\mu}$, meaningful search for resonant production of sgoldstino starts at integrated luminosity of $100 \mathrm{nb}^{-1}$, while integrated luminosity of up to $100 \mathrm{fb}^{-1}$ is required to fully explore the parameter space.

Sgoldstino with energy $E_{P}$ in laboratory frame flies, before its decay, a distance of about

$$
l_{P}=\frac{E_{P}}{m_{P}} \cdot(0.5 \mu \mathrm{m}-0.74 \mathrm{~cm}) .
$$

Thus, there is an opportunity to resolve sgoldstino vertices.

Let us now discuss briefly exotic models with hierarchy $A_{e} \gg A_{\mu}, M_{\gamma \gamma}$, where sgoldstino-electron coupling gets enhanced as compared to models with the case $A_{e} \sim A_{\mu} \sim M_{\gamma \gamma}$. First, $e^{+} e^{-}$decay mode of sgoldstino never becomes dominant: for sgoldstino with mass $m_{P}=214.3 \mathrm{MeV}$ the bound (11) on its lifetime gives unacceptably low scales of supersymmetry breaking, $\sqrt{F} \lesssim 200 \mathrm{GeV}$ even in unitarity limit $A_{e} \simeq \sqrt{F}$. Second, upper limit on $\operatorname{Br}\left(P \rightarrow e^{+} e^{-}\right)$is achieved for $A_{e}$ approaching unitarity limit $A_{e} \simeq \sqrt{F}$. Then, in models where sgoldstino decays dominantly into photon pairs, the upper bounds on $\operatorname{Br}\left(P \rightarrow e^{+} e^{-}\right)$are within $7 \cdot 10^{-3}-1 \cdot 10^{-6}$ for $\sqrt{F}=$ $2.5-60 \mathrm{TeV}$. In models with sgoldstino decaying mostly into $\mu^{+} \mu^{-}$-pair, the upper bounds on $\operatorname{Br}\left(P \rightarrow e^{+} e^{-}\right)$are between $0.7 \%$ and $1.4 \cdot 10^{-4}$ for $\sqrt{F}=2.5-60 \mathrm{TeV}$.

Thus, search for sgoldstino in the decay channel $P \rightarrow e^{+} e^{-}$is not promising even for $A_{e} \gg A_{\mu}, M_{\gamma \gamma}$. However, the cross section of the resonance production (23) is considerably enhanced for $A_{e} \gg A_{\mu}, M_{\gamma \gamma}$ as compared to the conservative case $A_{e} \sim A_{\mu} \sim M_{\gamma \gamma}$, so in this case sgoldstino would show up at relatively low integrated luminosity (of order $1 \mathrm{nb}^{-1}$ ). 


\section{Conclusions}

To conclude, we have identified the relevant region of parameter space of models with light sgoldstino, which can be responsible for anomalous events reported by HyperCP collaboration. We have estimated sgoldstino couplings and have predicted rates of rare three-body kaon decays and sgoldstino production in $e^{+} e^{-}$collisions. Sgoldstino may be searched for in $\mu^{+} \mu^{-}$and $\gamma \gamma$ decay channels, while $e^{+} e^{-}$channel never dominates. Experimental tests of our predictions will confirm or rule out sgoldstino explanation of HyperCP results.

The special features of the supersymmetric models, capable of explaining HyperCP results as evidence for sgoldstino, are: $(i)$ low energy scale of supersymmetry breaking in the underlying theory $(\sqrt{F} \sim 2.5-60 \mathrm{TeV})$, (ii) parity conservation (left-right entries in squark squared mass matrices make a hermitian matrix, $\left.\left(\tilde{m}^{(L R) 2}\right)^{\dagger}=\tilde{m}^{(L R) 2}\right)$, (iii) tiny flavor violation associated with $\tilde{m}_{\mathrm{D}, 12}^{(L R) 2}$ entry of squark squared mass matrix $\left(\tilde{m}_{\mathrm{D}, 12}^{(L R)} \lesssim 12 \mathrm{MeV}-1.4 \mathrm{GeV}\right.$ for $\sqrt{F}=0.5-60 \mathrm{TeV}$ and sgoldstino decaying mostly into $\mu^{+} \mu^{-}$; these bounds scale as $\operatorname{Br}^{-1 / 4}\left(P \rightarrow \mu^{+} \mu^{-}\right)$and become larger by an order of magnitude with smaller but still natural $A_{\mu} \simeq \alpha_{2} \cdot M_{\gamma \gamma}$ ). The latter feature implies either fine-tuning, or special flavor structure of MSSM soft terms preventing strong flavor violation.

We are indebted to D. Kaplan and M. Longo for extremely useful correspondence. We thank M. Danilov, V. Kekelidze, Yu. Kudenko, S. Kulagin, V. Obraztsov, P. Pakhlov and A. Skrinsky for stimulating discussions. This work was supported in part by the Russian Foundation for Basic Research grant 05-02-17363 and by the grant of the President of the Russian Federation NS-2184.2003.2. The work of D.G. was also supported in part by the Russian Foundation for Basic Research grant 04-02-17448, by the grant of the Russian Science Support Foundation and by the fellowship of the "Dynasty" Foundation (awarded by the Scientific board of ICFPM).

Note added. After the first version of this paper, Refs. [20, 21] were posted in arXives. We agree with Refs. [20,21] (as well as with Ref. [15]) that scalar $X \equiv S$ in (1) is inconsistent with other data, while pseudoscalar $X \equiv P$ is viable (it is pointed out in Ref. [20] that axial-vector $X \equiv A$ is another consistent possibility). The estimates given in Ref. [20], their Eq. (23), and in Ref. [21], Eq. (8), are also (almost) in agreement with our 
Eq. (9). Another overlap with Ref. [20, 21] is the possibility to search for $P$ in the decay $K_{L}^{0} \rightarrow \pi \pi P\left(P \rightarrow \mu^{+} \mu^{-}\right)$and their results in the case of real sgoldstino-quarks coupling constant are in numerical agreement with our estimates (16),(17).

\section{Appendix A}

The part of chiral lagrangian relevant for $K_{L, S} \rightarrow \pi \pi P$ decays occurring due to coupling (5), reads

$$
\mathcal{L}=\frac{f^{2}}{8} \operatorname{Tr}\left[\partial_{\mu} U^{\dagger} \partial_{\mu} U\right]+\frac{1}{4} B_{0} f^{2} \operatorname{Tr}\left[\chi U^{\dagger}+\chi^{\dagger} U\right]
$$

with $f=130 \mathrm{MeV}, B_{0}=m_{\mathrm{K}}^{2} /\left(m_{s}+m_{d}\right)$ and

$$
\begin{aligned}
U=\mathrm{e}^{i \frac{2}{f} \Phi}, \quad \Phi=\left(\begin{array}{ccc}
\frac{1}{\sqrt{2}} \pi^{0} & \pi^{+} & 0 \\
\pi^{-} & -\frac{1}{\sqrt{2}} \pi^{0} & K^{0} \\
0 & K^{0} & 0
\end{array}\right), \\
\chi B_{0} \hat{M}=\operatorname{diag}\left(m_{\pi}^{2}, m_{\pi}^{2}, 2 m_{\mathrm{K}}^{2}-m_{\pi}^{2}\right), \\
\hat{H}=\left(\begin{array}{ccc}
0 & 0 & 0 \\
0 & 0 & h_{12}^{(D)} \\
0 & h_{12}^{(D) *} & 0
\end{array}\right)
\end{aligned}
$$

where isospin symmetry violation due to different masses of up- and downquark is neglected. 
To the leading order in momenta one has the following interaction terms

$$
\begin{aligned}
\mathcal{L} & =\mathcal{L}_{4}^{k i n}+\mathcal{L}_{4}^{\text {pot }}+\mathcal{L}_{2 P}+\mathcal{L}_{4 P}, \\
\mathcal{L}_{4}^{\text {kin }} & =\frac{1}{3 f^{2}}\left(\bar{K}^{0} \partial_{\mu} K^{0}-K^{0} \partial_{\mu} \bar{K}^{0}\right)\left(\pi^{+} \partial_{\mu} \pi^{-}-\pi^{-} \partial_{\mu} \pi^{+}\right) \\
& -\frac{1}{3 f^{2}}\left(\bar{K}^{0} \partial_{\mu} \pi^{-}-\pi^{-} \partial_{\mu} \bar{K}^{0}\right)\left(K^{0} \partial_{\mu} \pi^{+}-\pi^{+} \partial_{\mu} K^{0}\right) \\
& -\frac{1}{6 f^{2}}\left(\bar{K}^{0} \partial_{\mu} \pi^{0}-\pi^{0} \partial_{\mu} \bar{K}^{0}\right)\left(K^{0} \partial_{\mu} \pi^{0}-\pi^{0} \partial_{\mu} K^{0}\right), \\
\mathcal{L}_{4}^{\text {pot }} & =\frac{m_{\mathrm{K}}^{2}+m_{\pi}^{2}}{3 f^{2}} K^{0} \bar{K}^{0}\left(\pi^{+} \pi^{-}+\frac{1}{2} \pi^{0} \pi^{0}\right), \\
\mathcal{L}_{2 P} & =B_{0} f\left(K^{0} h_{12}^{(D) *}+\bar{K}^{0} h_{12}^{(D)}\right) \cdot P, \\
\mathcal{L}_{4 P} & =-\frac{2 B_{0}}{3 f}\left(\bar{K}^{0} h_{12}^{(D)}+K^{0} h_{12}^{(D) *}\right)\left(\pi^{+} \pi^{-}+\frac{1}{2} \pi^{0} \pi^{0}\right) \cdot P .
\end{aligned}
$$

One can rewrite the lagrangian in terms of mass eigenvalues of the neutral kaon system,

$$
K_{L}^{0} \equiv \frac{1}{\sqrt{2}}\left(\bar{K}_{0}+K_{0}\right), \quad K_{S}^{0} \equiv \frac{i}{\sqrt{2}}\left(\bar{K}_{0}-K_{0}\right) .
$$



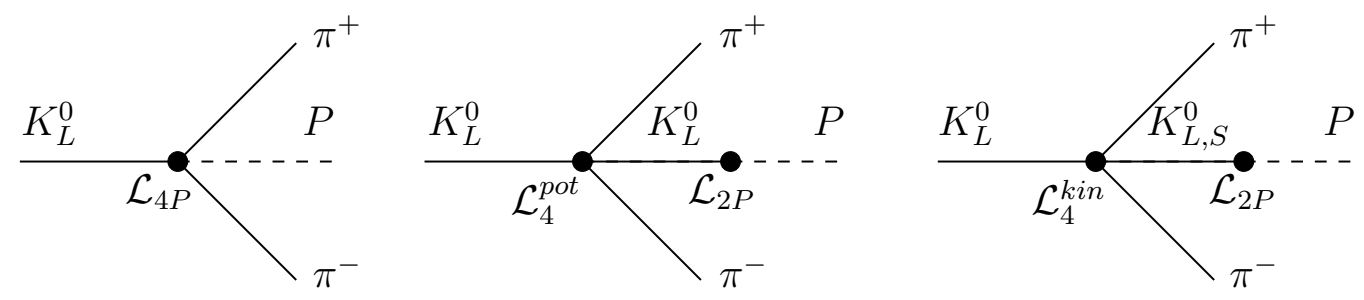

Figure 2: Diagrams contributing to $K_{L}^{0} \rightarrow \pi^{+} \pi^{-} P$ decay.

Then

$$
\begin{aligned}
\mathcal{L}_{2 P} & =\sqrt{2} B_{0} f\left(K_{L}^{0} \operatorname{Re} h_{12}^{(D)}+K_{S}^{0} \operatorname{Im} h_{12}^{(D)}\right) \cdot P \\
\mathcal{L}_{4 P} & =-\frac{2 \sqrt{2} B_{0}}{3 f}\left(K_{L}^{0} \operatorname{Re} h_{12}^{(D)}+K_{S}^{0} \operatorname{Im} h_{12}^{(D)}\right)\left(\pi^{+} \pi^{-}+\frac{1}{2} \pi^{0} \pi^{0}\right) \cdot P \\
\mathcal{L}_{4}^{p o t} & =\frac{m_{\mathrm{K}}^{2}+m_{\pi}^{2}}{6 f^{2}}\left(K_{L}^{02}+K_{S}^{02}\right)\left(\pi^{+} \pi^{-}+\frac{1}{2} \pi^{0} \pi^{0}\right) \\
\mathcal{L}_{4}^{k i n} & =-\frac{1}{6 f^{2}}\left(K_{L}^{0} \partial_{\mu} \pi^{-}-\pi^{-} \partial_{\mu} K_{L}^{0}\right)\left(K_{L}^{0} \partial_{\mu} \pi^{+}-\pi^{+} \partial_{\mu} K_{L}^{0}\right) \\
& -\frac{1}{6 f^{2}}\left(K_{S}^{0} \partial_{\mu} \pi^{-}-\pi^{-} \partial_{\mu} K_{S}^{0}\right)\left(K_{S}^{0} \partial_{\mu} \pi^{+}-\pi^{+} \partial_{\mu} K_{S}^{0}\right) \\
& -\frac{1}{12 f^{2}}\left(K_{L}^{0} \partial_{\mu} \pi^{0}-\pi^{0} \partial_{\mu} K_{L}^{0}\right)\left(K_{L}^{0} \partial_{\mu} \pi^{0}-\pi^{0} \partial_{\mu} K_{L}^{0}\right) \\
& -\frac{1}{12 f^{2}}\left(K_{S}^{0} \partial_{\mu} \pi^{0}-\pi^{0} \partial_{\mu} K_{S}^{0}\right)\left(K_{S}^{0} \partial_{\mu} \pi^{0}-\pi^{0} \partial_{\mu} K_{S}^{0}\right) \\
& +\frac{i}{2 f^{2}}\left(K_{L}^{0} \partial_{\mu} K_{S}^{0}-K_{S}^{0} \partial_{\mu} K_{L}^{0}\right)\left(\pi^{+} \partial_{\mu} \pi^{-}-\pi^{-} \partial_{\mu} \pi^{+}\right) .
\end{aligned}
$$

Hence, there are three contributions to the amplitude of $K_{L}^{0} \rightarrow \pi^{+} \pi^{-} P$ decay (see Fig. 2), 


$$
\begin{aligned}
\mathcal{L}_{4 P}: & -i \frac{2 \sqrt{2} B_{0}}{3 f} \operatorname{Re}_{12}^{(D)} \\
\mathcal{L}_{4}^{p o t}+\mathcal{L}_{2 P}: & -i \frac{2 \sqrt{2} B_{0}}{3 f} \operatorname{Re}_{12}^{(D)} \cdot \frac{m_{\mathrm{K}}^{2}+m_{\pi}^{2}}{2\left(m_{P}^{2}-m_{\mathrm{K}}^{2}\right)} \\
\mathcal{L}_{4}^{k i n}+\mathcal{L}_{2 P}: & i \frac{2 \sqrt{2} B_{0}}{3 f} \operatorname{Re}_{12}^{(D)} \cdot \frac{m_{P}^{2}+m_{\mathrm{K}}^{2}+2 m_{\pi}^{2}-3 m_{\pi^{+} \pi^{-}}^{2}}{4\left(m_{P}^{2}-m_{\mathrm{K}}^{2}\right)} \\
& -\frac{2 \sqrt{2} B_{0}}{f} \operatorname{Im} h_{12}^{(D)} \frac{m_{P \pi^{-}}^{2}-m_{P \pi^{+}}^{2}}{4\left(m_{P}^{2}-m_{\mathrm{K}}^{2}\right)},
\end{aligned}
$$

where $m_{i j}^{2} \equiv\left(p_{i}+p_{j}\right)^{2}$. The total amplitude to the leading order in momenta equals

$$
\begin{aligned}
\mathcal{M}\left(K_{L}^{0} \rightarrow \pi^{+} \pi^{-} P\right) & =-i \frac{2 \sqrt{2} B_{0}}{f} \operatorname{Re} h_{12}^{(D)} \cdot \frac{m_{P}^{2}-m_{\mathrm{K}}^{2}+m_{\pi^{+} \pi^{-}}^{2}}{4\left(m_{P}^{2}-m_{\mathrm{K}}^{2}\right)} \\
& -\frac{2 \sqrt{2} B_{0}}{f} \operatorname{Im} h_{12}^{(D)} \frac{m_{P \pi^{-}}^{2}-m_{P \pi^{+}}^{2}}{4\left(m_{P}^{2}-m_{\mathrm{K}}^{2}\right)}
\end{aligned}
$$

The last term is the analogue of the exchange interaction.

One has similar expressions for $K_{L}^{0} \rightarrow \pi^{0} \pi^{0} P$ decay,

$$
\begin{aligned}
\mathcal{L}_{4 P} & : \quad-i \frac{2 \sqrt{2} B_{0}}{3 f} \operatorname{Re}_{12}^{(D)} \\
\mathcal{L}_{4}^{p o t}+\mathcal{L}_{2 P}: & -i \frac{2 \sqrt{2} B_{0}}{3 f} \operatorname{Re}_{12}^{(D)} \cdot \frac{m_{\mathrm{K}}^{2}+m_{\pi}^{2}}{2\left(m_{P}^{2}-m_{\mathrm{K}}^{2}\right)} \\
\mathcal{L}_{4}^{k i n}+\mathcal{L}_{2 P}: & i \frac{2 \sqrt{2} B_{0}}{3 f} \operatorname{Re}_{12}^{(D)} \cdot \frac{m_{P}^{2}+m_{\mathrm{K}}^{2}+2 m_{\pi}^{2}-3 m_{\pi^{0} \pi^{0}}^{2}}{4\left(m_{P}^{2}-m_{\mathrm{K}}^{2}\right)}
\end{aligned}
$$

Note that the identity of final neutral pions implies the absence of the term proportional to the imaginary part of $h_{12}^{(D)}$, unlike the case of $K_{L}^{0} \rightarrow \pi^{+} \pi^{-} P$ decay. The total amplitude to the leading order in momenta equals

$$
\mathcal{M}\left(K_{L}^{0} \rightarrow \pi^{0} \pi^{0} P\right)=-i \frac{2 \sqrt{2} B_{0}}{f} \operatorname{Re} h_{12}^{(D)} \cdot \frac{m_{P}^{2}-m_{\mathrm{K}}^{2}+m_{\pi^{0} \pi^{0}}^{2}}{4\left(m_{P}^{2}-m_{\mathrm{K}}^{2}\right)} .
$$

The same expressions are valid for $K_{S}^{0}$ with the only replacement $\operatorname{Re} \leftrightarrow$ Im. 


\section{References}

[1] H. Park et al. [HyperCP Collaboration], Phys. Rev. Lett. 94, 021801 (2005).

[2] X. G. He, J. Tandean and G. Valencia, "The decay Sigma $+\rightarrow$ p l+ lwithin the standard model," arXiv:hep-ph/0506067.

[3] D. S. Gorbunov and V. A. Rubakov, Phys. Rev. D 64, 054008 (2001).

[4] http://www.fnal.gov/pub/news05/HyperCP.html

[5] J. Ellis, K. Enqvist, D. Nanopoulos, Phys. Lett. B147 (1984) 99; J. Ellis, K. Enqvist, D. Nanopoulos, Phys. Lett. B151 (1985) 357.

[6] T. Bhattacharya, P. Roy, Phys. Rev. D38 (1988) 2284.

[7] G. Giudice, R. Rattazzi, , Phys. Rep. 322 (1999) 419; S. L. Dubovsky, D. S. Gorbunov and S. V. Troitsky, Phys. Usp. 42, 623 (1999) [Usp. Fiz. Nauk 169, 705 (1999)].

[8] T. Bhattacharya, P. Roy, Phys. Lett. B206 (1988) 655.

[9] D. S. Gorbunov and A. V. Semenov, "CompHEP package with light gravitino and sgoldstinos," arXiv:hep-ph/0111291.

[10] D. S. Gorbunov, Nucl. Phys. B 602, 213 (2001).

[11] R. Kuchimanchi, Phys. Rev. Lett. 76 (1996) 3486; R. N. Mohapatra and A. Rasin, Phys. Rev. Lett. 76 (1996) 3490; R. N. Mohapatra and A. Rasin, Phys. Rev. D54 (1996) 5835; R. N. Mohapatra, A. Rasin and G. Senjanovic, Phys. Rev. Lett. 79 (1997) 4744.

[12] D. S. Gorbunov, "Hyperon physics with light sgoldstino", in Proc. of the 13th Int. Sem. "QUARKS'2004", May 24-30, 2005, Eds. D. G. Levkov, V. A. Matveev, V. A. Rubakov, INR RAS, Vol.I, 472-481.

[13] A. Brignole, F. Feruglio and F. Zwirner, Nucl. Phys. B 501 (1997) 332.

[14] S. Eidelman et al. [Particle Data Group], Phys. Lett. B 592, 1 (2004).

[15] L. Bellantoni [KTEV Collaboration], "Recent results from KTeV," arXiv:hep-ex/0506017. 
[16] A. Alavi-Harati et al. [KTEV Collaboration], Phys. Rev. Lett. 84, 5279 (2000).

[17] R. A. Burnstein et al. [HyperCP Collaboration], Nucl. Instrum. Meth. A 541, 516 (2005).

[18] G. Colangelo, J. Gasser and H. Leutwyler, Phys. Lett. B 488, 261 (2000).

[19] S. Pislak et al., Phys. Rev. D 67, 072004 (2003).

[20] X. G. He, J. Tandean and G. Valencia, arXiv:hep-ph/0509041.

[21] N. G. Deshpande, G. Eilam and J. Jiang, arXiv:hep-ph/0509081. 